\title{
Electric wind generation by nanosecond repetitively pulsed microplasmas
}

\author{
Thomas Orrièreł, Éric Moreau, David Z. Pai \\ Institut PPRIME - UPR 3346 CNRS - Université de Poitiers - ISAE/ENSMA \\ Futuroscope Chasseneuil Cedex, France \\ E-mail: thomas.orriere@univ-poitiers.fr, david.pai@univ-poitiers.fr \\ March 2019
}

\begin{abstract}
.
A nanosecond repetitively pulsed microplasma generated between two tungsten electrodes in a pin-to-pin configuration is used as a supply of charged species to produce an electrohydrodynamic phenomenon when placed near a DCbiased electrode. We employed high-frequency particle image velocimetry in order to study the produced flow. The generated flow velocities are around $2 \mathrm{~m} \mathrm{~s}^{-1}$ with a deposited average power on the order of $1 \mathrm{~W}$. The main characteristics of this flow and the impact of the frequency and the DC field are discussed. In the end, we further explain the impact of each microplasma event on flow modification.
\end{abstract}

\section{Introduction}

Electrohydrodynamic (EHD) phenomena involving electrical discharges in air at atmospheric pressure are useful in many fields such as flow control (Moreau 2007, Corke et al. 2010), electrostatic precipitation (Zouzou \& Moreau 2011), combustion (Lawton \& Weinberg 1969, Lacoste et al. 2017), food drying (Martynenko \& Kudra 2016) and thrusters (Christenson \& Moller 1967, Moreau et al. 2013, Xu et al. 2018). $\ddagger$ current affiliation: Clean Combustion Research Center, King Abdullah University of Science and Technology (KAUST), Thuwal 23955-6900, Saudi Arabia, thomas.orriere@kaust.edu.sa 


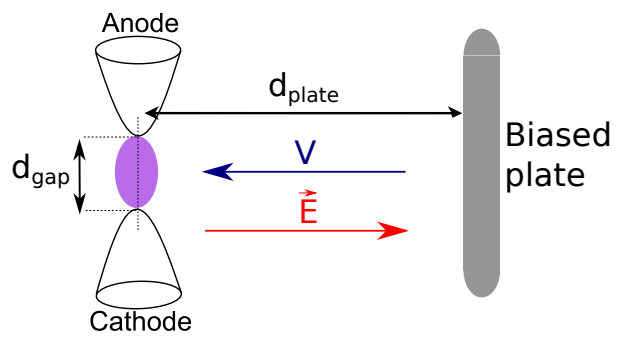

Figure 1. Example of schematic diagram of a microplasma geometry to produce ionic wind. The distance $d_{\text {gap }}$ have to be smaller than the mm-scale and the distance $d_{\text {plate }}$ is longer than the cm-scale.

Furthermore, ionic or electric wind can be an important aspect of widely used discharge setups such as plasma jets (Robert et al. 2014, Darny et al. 2017).

The underlying theory of EHD flows was established several decades ago (Robinson 1961, Robinson 1962) : charged species drift due to an applied electric field and transfer momentum through collisions to the surrounding neutral species. However, in contrast with the simplicity of the phenomenon, the underlying physics are very complex due to the combination of fluid mechanics, physics of plasmas and chemistry in air (Boeuf \& Pitchford 2005, Nishida et al. 2014, Moreau et al. 2018).

In all the research areas listed above, corona discharges and dielectric barrier discharges (DBDs) are almost exclusively used. In flow control, DBDs are widely used thanks to their stability and ease of implementation due to their self-limiting characteristics preventing them to turn into arc discharges (Kogelschatz et al. 1997).

Usually the design of an actuator consists of predicting the flow pattern from the electric field profile and electrode geometry. However, the discharge regime and structure are also closely connected to the electric field and to the shape of the electrodes. Thus, the flow produced by a plasma actuator is not easily predictable and cannot be modified in a straightforward manner by changing the geometry (Moreau 2007, Corke et al. 2010). 
A simple solution to address this problem could be the separation of the production of the charged species and their transport. Here, we suggest to use a microdischarge as a charge supplier and the use one or several electrodes to drive the charges via the electric field shape. The figure 1 shows an example of such a system composed by a microplasma generated between two pin electrodes and a plate biased by a DC voltage.

A similar idea with five electrodes has already been pursued with corona discharges (Colas et al. 2010), but the ion production and ion drift were not fully decoupled. Later, several studies (Tirumala \& Go 2011, Johnson et al. 2015) presented similar three-electrode geometry but focused on miniaturizing the plasma down the mm-scale for convective heat transfer purposes.

Concerning the DBDs, sliding discharge actuators involving three electrodes have also been designed (Moreau et al. 2008, Bayoda et al. 2015, Nishida et al. 2017) with the purpose of elongating the discharge rather than separating the production and transport of the charged species.

Microplasmas are generated by confining a discharge to the micrometer scale in order to enhance surface interaction. In the past, microplasmas have shown interesting properties for the applications, such as high electron temperature, electron density, low gas temperature and good stability (Becker et al. 2006, Mariotti \& Sankaran 2010). By combining microplasmas with nanosecond repetitively pulsed (NRP) discharges, electron number density and temperature can be further increased (Zhu et al. 2012, Huang et al. 2013, Orrière et al. 2018). NRP discharges in the spark regime with mm-scale gap distances have already been successfully applied in plasma assisted combustion due to their thermal, chemical and acoustic properties (Pilla et al. 2006, Pancheshnyi et al. 2006, Xu et al. 2015, Bölke et al. 2018). 
Many of the above properties of mm-scale NRP sparks may be preserved by microplasmas, which may also possess enhanced plasma properties. In air at atmospheric pressure, a very high ionization fraction of about 0.5 has already been reached (Orrière et al. 2018). Furthermore the use of nanosecond pulsing $(\approx 10 \mathrm{~ns}$ ) with a low duty cycle decouples the interaction between the large-scale electric field from that of the microplasma.

Another issue concerns the chemistry of the discharges producing electric wind. According to (Johnson \& Go 2017), humidity and ozone production are of particular concern. Methods have already been found to reduce ozone production for corona discharges, by changing the geometry (Chen \& Davidson 2002, Yehia \& Mizuno 2005) or heating the active electrode (Awad \& Castle 1975). Humidity has a great impact on the discharge dynamics, chemistry and consequently produced ionic wind for corona discharges. As far as we know, there are no clear options to reduce the impact of humidity without employing a dehumidifier, and ozone reduction requires constraints upon the geometry.

The use of an NRP spark discharge could reduce the impact of humidity on plasma properties. A comparative study in pure $\mathrm{N}_{2}$ and in $\mathrm{N}_{2}$ with $0.9 \% \mathrm{H}_{2} \mathrm{O}$ has already been conducted at atmospheric pressure for a NRP discharge at the mm scale (Van der Horst et al. 2012). The spark regime showed no difference in term of discharge form and electron number density. Fast gas heating was observed only with the addition of water vapor, but this is also expected because oxygen is present, as in the case of air (Rusterholtz et al. 2013).

Furthermore, ozone production is expected to be low when the electric field is high (greater than 500 Td) (Plank et al. 2014) or when species which can easily destroy $O_{3}$ are produced, e.g. $N$ atoms or $N O$. Such species are expected to be produced in high 
densities in this type of discharge, as modeled by (Popov 2016) and suggested by the experimental results of (Orrière et al. 2018).

In this paper, we study the applicability of NRP microplasmas to produce EHD phenomena. In particular, we would like to separate the mechanisms of charge production and charge drift by using NRP microplasmas.

If decoupling is possible, then the microplasma can be treated as an ion source for designing EHD devices. EHD models already exist that treat the discharge as a simplifed ion source (Boeuf \& Pitchford 2005, Go et al. 2007). Ideally, a full numerical model of the NRP microplasma would provide the correct input for the ion source. Such a model does not yet exist, but the literature on related NRP spark discharges (Rusterholtz et al. 2013, Popov 2016) and experimental on NRP microplasmas (Orrière et al. 2018) make it possible to approximate the amount of ionization and recombination in the discharge gap. Additional advantages could include the reduction of ozone production and sensitivity to humidity, although these subjects are outside the scope of this study.

This paper is organized as follows, in section 2 we will present the reactor geometry and the experimental methods. We used passive probes to characterize the voltage, current and power of the microplasma and particle image velocimetry (PIV) and Schlieren photography to characterize the flow. We will present and discuss the results in section 3. The electrical characterization will be discussed first in section 3.1. Then we will present velocity fields obtain by PIV measurements in section 3.2, under standards conditions previously defined at high repetition frequency $\left(F_{N R P}=8 \mathrm{kHz}\right)$. The impact of the voltage applied to the third electrode will be shown in section 3.3. In section 3.4, we will show time-resolved snapshots of the initial velocity fields when the microplasma is turned on with the flow initially at rest. We will also further study 


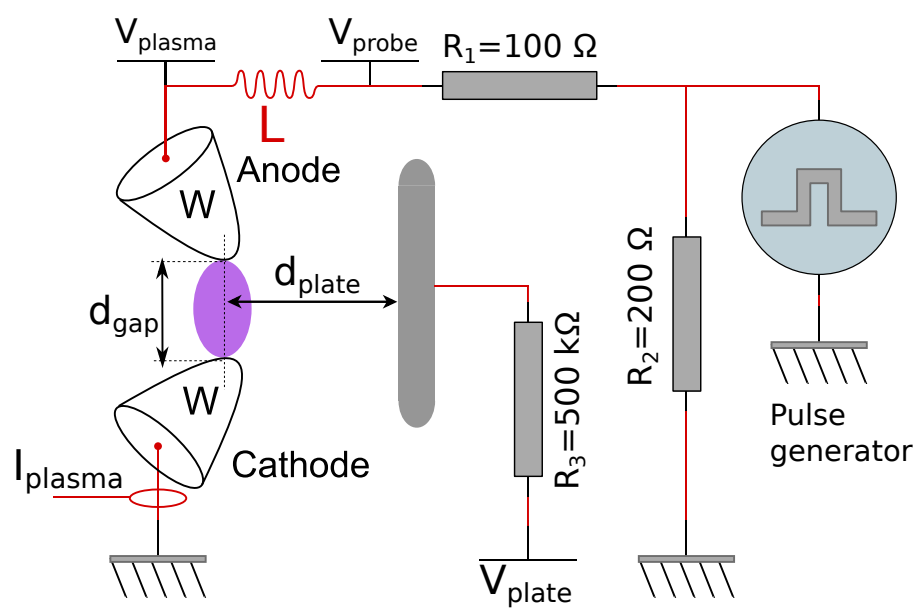

Figure 2. Schematic diagram of the electrical circuit and setup

the transient phenomenon by using a low repetition frequency $F_{N R P}=100 \mathrm{~Hz}$.

\section{Experimental setup}

\subsection{Electrode configuration and electrical measurement system}

NRP micro-discharges were generated between two tungsten electrodes sharpened to a radius of curvature of $280 \mu \mathrm{m}$ in air seeded by dielectric oil droplets at atmospheric pressure. The entire experiment was placed inside an unsealed (but closed) PMMA tank. High voltage pulses were applied across the $200 \mu \mathrm{m}$ gap $\left(d_{\text {gap }}\right)$ with a $6 \mathrm{kV}$ amplitude and a $15 \mathrm{~ns}$ duration. As shown on figure 2, a nanosecond pulsed generator (FID Technology FPG 40-30 NK) transmitted pulses with a $75 \Omega$ impedance line. Two resistances, namely $R_{1}$ and $R_{2}$ (Genvolt, RES200 serie) achieved a rough impedance matching allowing the release of $>80 \%$ of the energy in open or a short circuit load conditions. Two passive probes (Lecroy PPE $6 \mathrm{kV}, 400 \mathrm{MHz}$ bandwidth and Bergoz CT-D5.0, $4.8 \mathrm{kHz}-400 \mathrm{MHz}$ bandwidth) measured the voltage applied over

the microreactor $V_{\text {probe }}$ and the current through the discharge $I_{\text {plasma }}$. An oscilloscope 
(Lecroy Waverunner $204 \mathrm{MXI}, 2 \mathrm{GHz}$ bandwidth, $5 \times 10^{12} \mathrm{~s}^{-1}$ sampling rate) recorded all the signals. Due to the high current $(\approx 40 \mathrm{~A})$, two attenuators were placed on the current probe terminal $(-20 \mathrm{~dB}$, Telegartner J011006A0837). The voltage has been corrected to take into account the inductance of the cables symbolized by $L$ on figure 2 . This method has already been introduced in a previous study (Orrière et al. 2018) as well as the calculation of the energy per pulse.

The electrodes were inclined at an angle of $45^{\circ}$, as shown on figure 3 , both in order to increase the electric field and to gain optical access to the microplasma region. A DC power supply (DEL Electronics, Model RLPS 40-150) was connected to the third electrode via a high voltage resistance $R_{3}$ (Powertron HTE152, $500 \mathrm{k} \Omega$ ) in order to prevent short-circuits. The third electrode was a $8 \mathrm{~cm}$ diameter stainless steel plate with rounded edges and was placed at a distance $d_{\text {plate }}$ away from the microplasma reactor. In all cases, we used $d_{\text {plate }}=40 \mathrm{~mm}$ and $d_{\text {gap }}=200 \mu \mathrm{m}$.

\subsection{PIV measurements}

Figure 3 shows a schematic diagram of the geometry. Two planes of the flow were studied: the XY plane parallel to the axis of the discharge and the perpendicular XZ plane slicing through the middle of the discharge (figure 3). To record both XY and $\mathrm{XZ}$ planes, the plate, the camera and the laser sheet was not moved, but we rotated the reactor around the $\mathrm{X}$ axis. Consequently, the $\mathrm{Y}$ axis is vertical when we recorded the $\mathrm{XY}$ plane, and the $\mathrm{Z}$ axis is vertical when we recorded the $\mathrm{XZ}$ plane. The $0.122 \mathrm{~m}^{3}$ PMMA tank was seeded by droplets of Ondina Oil 915 with a size between $0.2 \mu \mathrm{m}$ $1 \mu \mathrm{m}$ provided by an aerosol generator (TOPAS ATM-200). A laser emitting at 532 nm (Nd:Yag Continuum Mesa) was shaped into a 1-mm thick laser sheet to illuminate the seeded particles. For PIV of the entire flow region between the microplasma and 


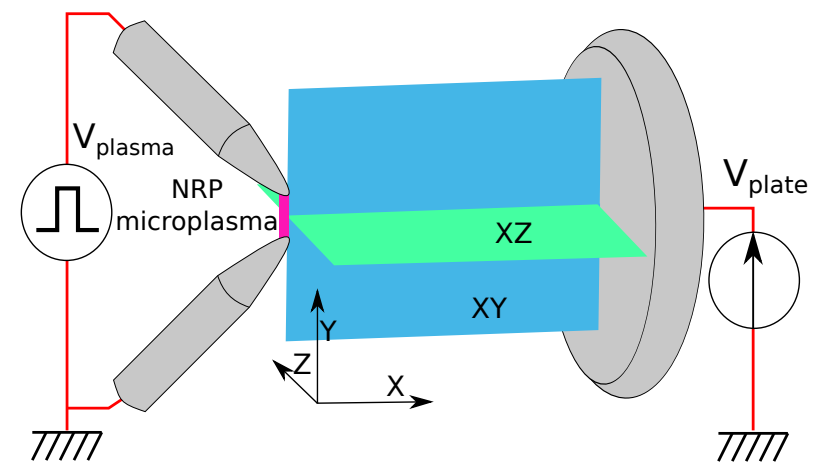

Figure 3. Schematic diagram of the geometry of the three electrode setup. The planes XY and XZ represent the laser sheet positions for PIV measurements.

the plate, a $1024 \times 1024$ pixel fast camera (Photron Fastcam SA-Z) equipped with a $60 \mathrm{~mm}$ objective (AF Micro-Nikkor $60 \mathrm{~mm}$ 1:2.8 D) recorded the particle images over a field of view of $43 \times 43 \mathrm{~mm}$.

For PIV zoomed in on the region closed to the microplasma, we replaced the $60 \mathrm{~mm}$ objective with a long-range microscope (Distamax by Infinity, Model K2). The field of view was then $5.5 \times 5.5 \mathrm{~mm}$ on the camera. The maximum frame rate of this system was $20 \mathrm{kHz}$. A trigger source (Stanford Research system DG645) synchronized all devices. Images were processed with the Davis 8 software using a double-pass algorithm where the field of view is divided into round interrogation windows that reduce in size with each pass (from $32 \times 32$ to $16 \times 16$ with $50 \%$ overlap), yielding a vector field spatial resolution of $329 \mu \mathrm{m}$ or $82 \mu \mathrm{m}$ for the zoomed experiment. In order to de-noise the snapshots, a two-dimensional median filter has been applied spatially with a kernel size of three points and then a median filter on five points temporally. 


\section{Results and discussion}

\subsection{Electrical characterization of the microplasma}

Figure 4 shows an example of typical electrical waveforms of the microplasma after applying pulses at a repetition frequency $F_{N R P}=8 \mathrm{kHz}$ during the PIV measurement. The impulsion starts at $t=0 \mathrm{~ns}$ and reaches a maximum of $3 \mathrm{kV}$ at $t=8 \mathrm{~ns}$. After spark breakdown, the conduction current flowing into the discharge increased up to $33 \mathrm{~A}$ within $4 \mathrm{~ns}$. Thus an energy of $\overline{U_{\text {plasma }}}=121 \pm 15 \mu \mathrm{J}$ is deposited in the microplasma.

The average power transferred in the discharge can then be calculated $P=$ $0.97 \mathrm{~W}$ while the peak power is around $20 \mathrm{~kW}$. No measurable effect of $V_{\text {plate }}$ has been observed in the range $V_{\text {plate }} \in[0 \mathrm{kV}, 20 \mathrm{kV}]$ on microplasma power consumption. However, at $V_{\text {plate }}=-14 \mathrm{kV}$, a time-averaged current smaller than $5 \mu \mathrm{A}$ flows from the third electrode positioned at $d_{\text {plate }}=40 \mathrm{~mm}$ to the ground through the DC power supply. This contribution of $0.07 \mathrm{~W}$ is minor in comparison to the microplasma but should be taken into account for accurate measurements.

In a previous study, the discharge was placed in open air without seeded particles (Orrière et al. 2018), and the ionization fraction was found to be very high $\left(1 \times 10^{19} \mathrm{~cm}^{3}\right)$ as well as the average reduced electric field $(660 \mathrm{Td})$. Interestingly, the average power deposited in the NRP microplasma is of the same order of magnitude as for the corona discharges $(0.1 \mathrm{~W}-1 \mathrm{~W})$ usually used to produce ionic wind (Johnson \& Go 2017). We will further see in the next sections that the flow velocities are also of the same order of magnitude. However, discharges characteristics are far different from those of a corona discharge since the energy density and the electric field during the breakdown are higher for NRP microplasmas. This should result in a better ion 


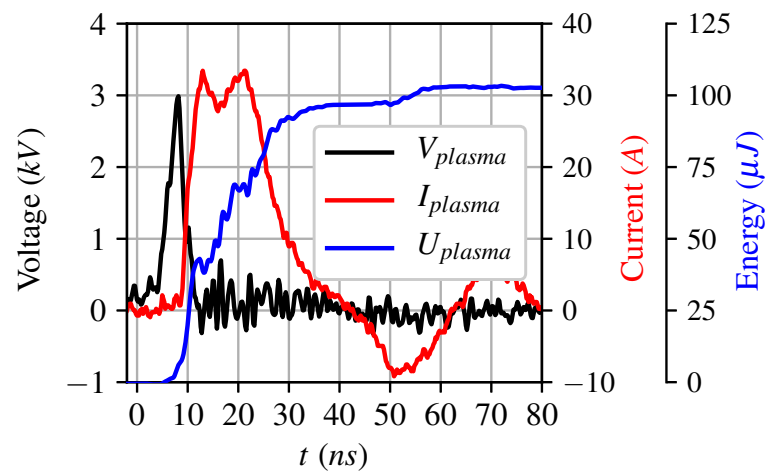

Figure 4. Single shot measurement of the applied voltage, current and deposited energy for $F_{N R P}=8 \mathrm{kHz}$.

production efficiency since the electric field is close to that of the optimal electric field around $1000 \mathrm{Td}$ for producing charged species (Macheret et al. 2006).

\subsection{Flow fields}

In this subsection, we show the PIV results and further explain the obtained velocity fields. Figure 5 shows the time-averaged velocity magnitude $\bar{U}$ and streamlines of the vector field in the XY (Figure $5(\mathrm{a})$ ) and XZ planes (Figure 5(b)) in the same conditions as the measurements shown in figure $4\left(V_{\text {plate }}=-14 \mathrm{kV}, F_{N R P}=8 \mathrm{kHz}\right.$, $\left.d_{\text {plate }}=40 \mathrm{~mm}\right)$. Each of these velocity fields is averaged over 6000 snapshots of the velocity recorded at a frequency $F_{P I V}=16.25 \mathrm{kHz}$ without any common trigger source.

In the two planes, we can observe a flow between the microplasma positioned at $x=y=z=0 \mathrm{~mm}$ and the plate at $x=d_{\text {plate }}=40 \mathrm{~mm}$. The central maximum flow velocity is $\overline{U_{x}}=1.5 \mathrm{~m} \mathrm{~s}^{-1}$. The flow velocity remains in the range of that reported for corona discharges (Johnson \& Go 2017), at similar measured power as reported in section 3.1. Higher velocities of about $8 \mathrm{~m} \mathrm{~s}^{-1}$ have been measured in corona discharges 


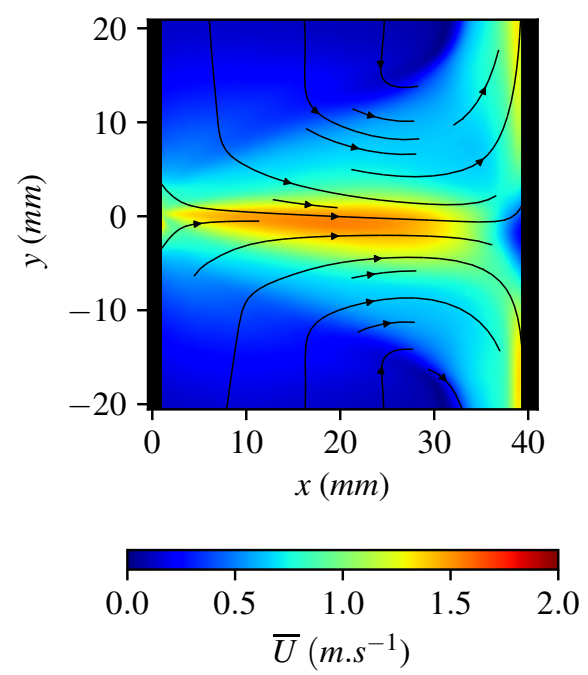

(a)

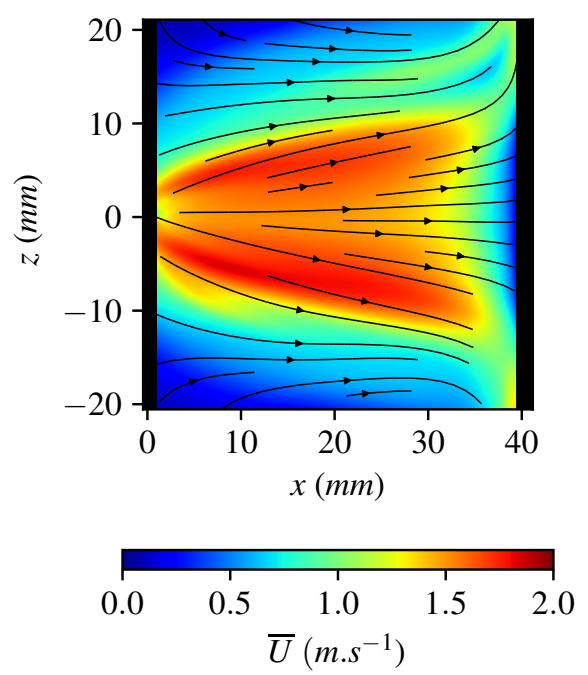

(b)

Figure 5. (a) Mean velocity field superimposed by streamlines of the vector field in the XY plane where the position of the microplasma is $x=y=z=0 \mathrm{~mm}$ and the position of the third plate electrode is $x=40 \mathrm{~mm}$ with $V_{\text {plate }}=-14 \mathrm{kV}$ (b) in the XZ plane

produced by applying a voltage of $16 \mathrm{kV}$ across a $25 \mathrm{~mm}$ gap distance in a pin to plane geometry using the same PIV method and setup as in this work (Moreau et al. 2018).

In EHD flows, as long as space charge exists, momentum is transferred to the fluid in the direction of the local electric field. The maximum flow velocity region can be positioned away from the discharge, and this flow velocity can be maintained even if shear exists as in real fluid flows. We see that the flow accelerates near the discharge up to $x=10 \mathrm{~mm}$ and then remains constant up to $x=30 \mathrm{~mm}$. This would imply that the EHD force exactly counters the viscous forces from $x=10 \mathrm{~mm}$ to $x=$ $30 \mathrm{~mm}$. The jet then splits up and down at the stagnation point where the velocity is nearly $0 \mathrm{~m} \mathrm{~s}^{-1}$ in the impingement region.

The velocity fields in the two planes are not the same due to the asymmetry of the configuration. In the XY plane, the maximum of the flow velocity is at the center of the jet, whereas in the $\mathrm{XZ}$ plane a decrease of the flow velocity is observed. This 
behavior can be due to the wake of the electrodes. Moreover, the presence of the discharge can enhance the wake effect over the electrodes, especially at high frequency $F_{N R P}$ (Seydou Moumouni 2016).

PIV is considered as an intrusive measurement because particles can act on the plasma physics and chemistry. Considering the electrical characteristics, the applied voltage increases by less than $20 \%$ because the breakdown delay is longer, allowing the voltage pulse to reach a higher value before truncation due to low plasma impedance. We observed no significant differences on the discharge current and energy when particles are added.

The reverse process is also possible. The main assumption of the PIV technique is that the seed particles track the fluid flow faithfully. Plasmas are known to greatly affect particle motion though charging effects and the presence of the electric field. To examine this potential problem, we used another flow diagnostic: Schlieren imaging which does not require particles. The Schlieren photographs are presented for several $d_{\text {gap }}$ distances in Appendix A. These images, performed in equivalent conditions as for figure 5 but without particles, prove that the flow exists in non-seeded air. Furthermore, the velocity reaches zero at the plate, as expected for fluid flow but not in the case of electrostatic precipitation (Defoort et al. 2018). A recent study of the ionic wind produced by a corona discharge has shown a strong agreement between time-resolved Schlieren photographs and PIV velocity fields (Moreau et al. 2019). Thus, the plasma does not affect the PIV results in a major way. We will further discuss this point in section 3.4. 


\section{3. $V_{\text {plate }}$ variation}

In this section we further investigate the influence of $V_{\text {plate }}$ on the flow velocity. Six $V_{\text {plate }}$ voltages have been tried from $-16 \mathrm{kV}$ to $16 \mathrm{kV}$. We report on figure $6(\mathrm{a})$ the axial velocity $U_{c}$, which represents the mean axial velocity at the center of the jet in the two planes $\left(U_{c}=\overline{U_{x}}(y=z=0)\right)$. A great dependance of the flow velocity on $\left|V_{\text {plate }}\right|$ is observed, thus further confirming flow production by ionic wind. No significant differences between the negative and the positive polarity of the plate are observed. It follows that imposing either a negative or a positive electric field attracts the opposite charged species to the plate.

This implies that either positive or negative ions produced by the microplasma contribute to the EHD force in about equal number. Also, the spatial separation of the regions of charge generation (microplasma) and charge transport (to the plate) has successfully separated the ions such that only ions of one polarity contributes to EHD. Otherwise the positive and negative ion contributions would cancel each other. To reduce any possible influence of the DC field on the microplasma, a positive $V_{\text {plate }}$ should be used to decrease the voltage difference between the high-voltage electrode of the microplasma and the plate, given that the pulse voltage is also positive.

Let us now consider the shape of the central axial velocity $U_{c}$. A plateau is observed for $x \in[10 \mathrm{~mm}, 30 \mathrm{mn}]$ and should be due to the equilibrium between forces which are moving the fluid and those which dissipate the inertial energy of the flow via viscosity. For the negative polarity, we show the velocity profiles at $x=d_{\text {plate }} / 2$ in both the XZ and XY plane on figure 6(b). The shape of the cross-sectional profile of the flow does not significantly change with applied voltage in either plane. A reasonable agreement between $U_{c}$ on the $\mathrm{XY}$ and $\mathrm{XZ}$ plane is observed for all potential $\left|V_{\text {plate }}\right|$. 


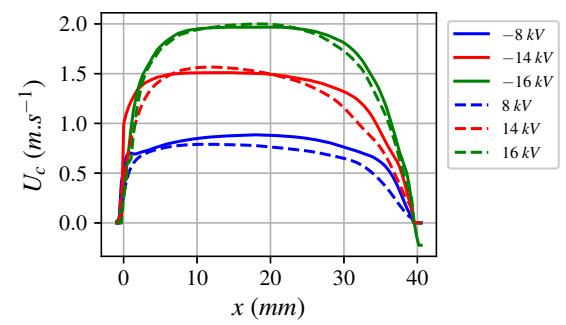

(a)

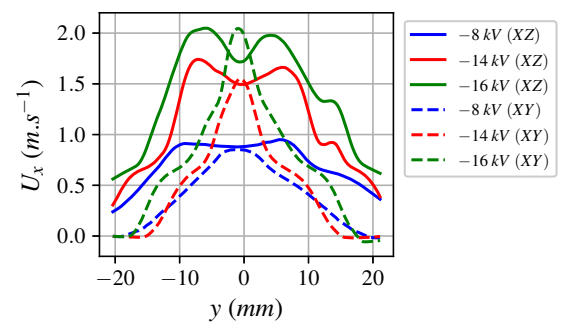

(b)

Figure 6. (a) Axial velocity $U_{c}$ along the $x$ axis $\left(U_{c}=\overline{U_{x}}(y=z=0 \mathrm{~mm})\right)$ with varying $V_{\text {plate }}, d_{\text {plate }}=40 \mathrm{~mm}$ and $F_{N R P}=8 \mathrm{kHz}$. Profiles are extracted from the XY plane. (b) Axial velocity profiles in the XZ and XY plane for $x=20 \mathrm{~mm}$ and $V_{\text {plate }} \in[-8 \mathrm{kV} ;-16 \mathrm{kV}]$.

\subsection{Transient phenomena}

In this section, we investigate the transient properties of EHD flows produced by NRP microplasmas in order to get a more detailed understanding of the EHD phenomenon.

Figure 7(a) is a series of time-resolved snapshots of the velocity magnitude in the $\mathrm{XZ}$ plane upon switching on the microplasma initially at $t=0 \mathrm{~ms}$. Figure 7 (b) shows the corresponding axial component at the center of the flow at the indicated time $t$. From figure $7(\mathrm{a})$, we can see that the flow studied in section 3.2 takes around $40 \mathrm{~ms}$ to be formed.

The XZ profile observed in figure 5(b), with the maximum flow velocity not positioned at the center of the jet, is seen at the very beginning of the series at $t$ $=3 \mathrm{~ms}$. Thereafter, the jet front continues to move towards the plate at a speed of about $2 \mathrm{~m} \mathrm{~s}^{-1}$ until impingement. The flow acceleration does not occur only with the movement of the jet front. A flow velocity around $0.5 \mathrm{~m} \mathrm{~s}^{-1}$ is observed downstream of the jet front at $t \in[3 \mathrm{~ms}, 22 \mathrm{~ms}]$, which is especially clear on figure $7(\mathrm{~b})$. 

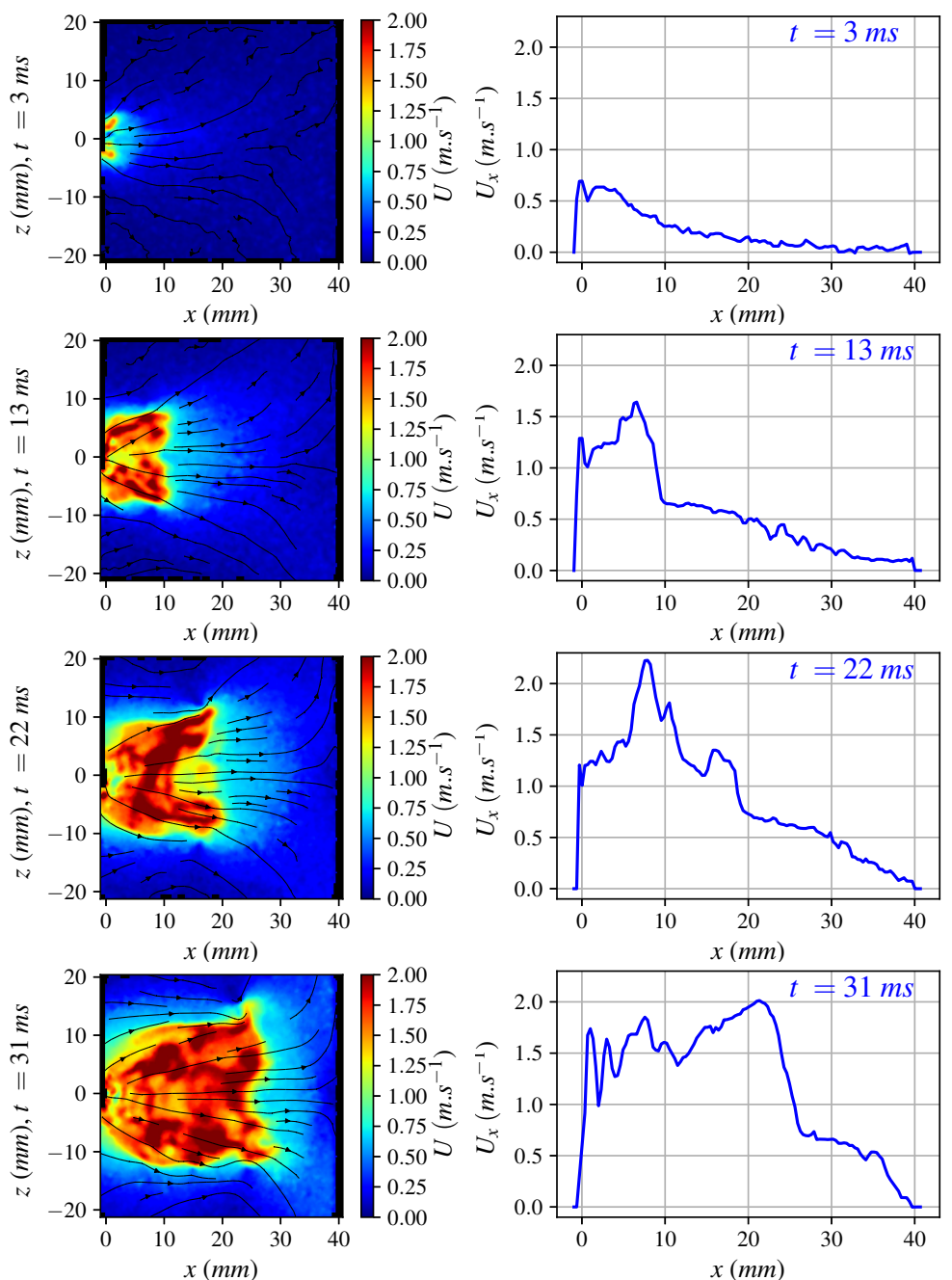

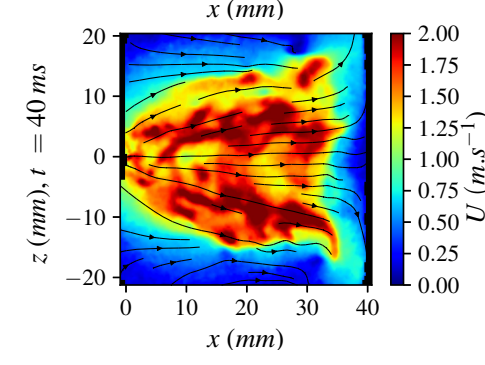

(a)

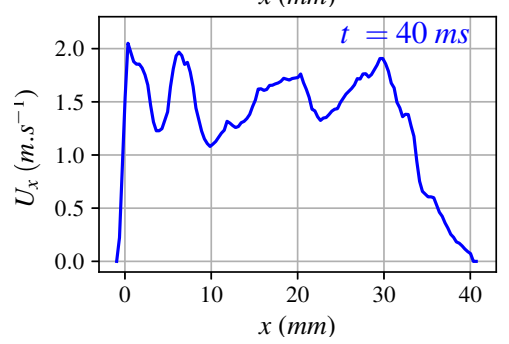

(b)

Figure 7. (a) Snapshots of the velocity magnitude after switching on the NRP microplasma at $t=0$ with the same experimental conditions as in section 3.2 $\left(V_{\text {plate }}=-14 \mathrm{kV}, F_{N R P}=8 \mathrm{kHz}, d_{\text {plate }}=40 \mathrm{~mm}\right),\left(\right.$ b) axial velocity $U_{x}$ for $y$ $=0 \mathrm{~mm}$ at different times after switching on the discharge. We show only the XZ plane. 
This first flow acceleration observable upon switching on the microplasma should not be due to seed particle precipitation. The contribution to the total velocity of a charged seed particle due to electrostatic drift is relatively high at low fluid velocity compared to high fluid velocity (like on figure 7 (a) and (b) at $t=3 \mathrm{~ms}$ ). However, in the early step at $t=3 \mathrm{~ms}$ or $t=13 \mathrm{~ms}$, we can see on figure $7(\mathrm{a})$ that the flow streamlines bypass parallel to the plate at $x=40 \mathrm{~mm}$. If the movement of particles were mainly due to precipitation, the streamlines would follow the electric field and terminate perpendicular to the plate. Furthermore, the axial velocity profile on figure 7(b) shows zero velocity of the flow near the plate even at $t>30 \mathrm{~ms}$, as discussed in section 3.2 for time-averaged flow fields.

The time-resolved velocity profiles of unsteady inertial jets also typically exhibit this first flow acceleration in front of the jet. Similar two-step accelerations have been seen in the experimental work of (Zong \& Kotsonis 2018) on a plasma synthetic jet. The high-velocity region of the jet displaces the fluid downstream due to conservation of mass flux, accelerating the flow in the downstream region. Thus, the jet front is never perfectly sharp. Therefore, the unsteady flow produced by the microplasma at $F_{N R P}=8 \mathrm{kHz}$ is similar to that of typical pulsed jets.

However, in our case, the flow is generated by the electric wind, and a substantial force can be applied where there is space charge. We may ask whether an impulse injection of EHD force due to the drift of the ions may also contribute to this first acceleration. Ions can drift much faster than the jet front: with an ion mobility of the expected ions in air between $2 \times 10^{-4} \mathrm{~m}^{2} \mathrm{~V}^{-1} \mathrm{~s}^{-1}$ and $3 \times 10^{-4} \mathrm{~m}^{2} \mathrm{~V}^{-1} \mathrm{~s}^{-1}$ (Radzig \& Smirnov 1985) and a mean electric field $E=0.35 \times 10^{6} \mathrm{Vm}^{-1}$ between the microplasma and the plate, the ion drift velocity should be $v_{i} \approx 80 \mathrm{~m} \mathrm{~s}^{-1}$. Therefore the ions can drift and transfer their momentum to the fluid very quickly. 


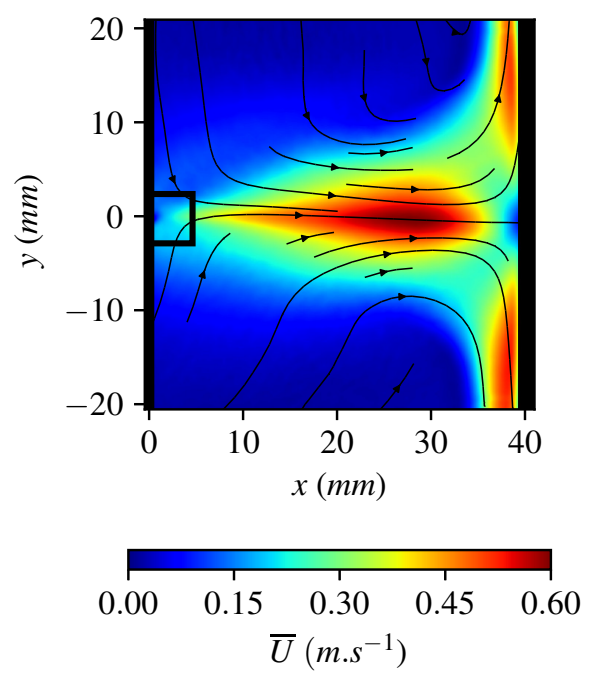

(a)

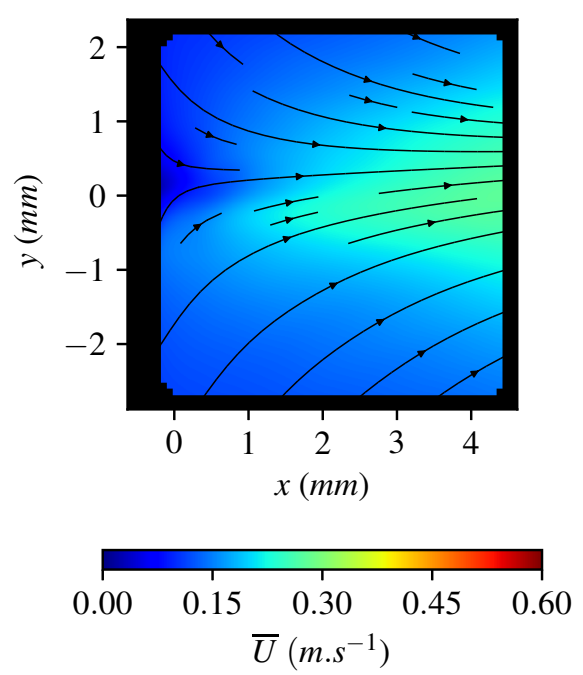

(b)

Figure 8. (a) Mean velocity field in the XY plane where the position of the microplasma is $x=y=z=0 \mathrm{~mm}$ and the position of the third plate electrode is $x=40 \mathrm{~mm}$ with $F_{N R P}=100 \mathrm{~Hz}$ and $V_{\text {plate }}=-14 \mathrm{kV}$. The black rectangle highlights the measured domain of figure (b). (b) Mean velocity field in the XY plane by using a higher magnification near the microplasma. A black rectangle on figure (a) highlights the limits of figure (b).

To investigate this possibility, we studied a case with $F_{N R P}=100 \mathrm{~Hz}$. A low frequency as $100 \mathrm{~Hz}$ allows us to understand the impact of each microplasma event upon the induced flow. Unfortunately, the discharge cannot be classified as a NRP discharge since the frequency is too low. Thus, the discharge should be very different with regard to memory effects between cases with $F_{N R P}=8 \mathrm{kHz}$ and $F_{N R P}=100 \mathrm{~Hz}$. However, we believe that the EHD properties of both discharges should be similar.

Figure $8(\mathrm{a})$ shows the velocity magnitude field in the $\mathrm{XY}$ plane for $F_{N R P}=$ $100 \mathrm{~Hz}$. The main structure remains the same as for figure 5, but we observe two differences: the velocity is lower (by a factor $\times 2.5$ ), and there is no plateau because the acceleration is maintained until $x=30 \mathrm{~mm}$. The counterbalancing viscous force is too low due to the very low generated velocity, allowing the flow to continue accelerating further downstream. 


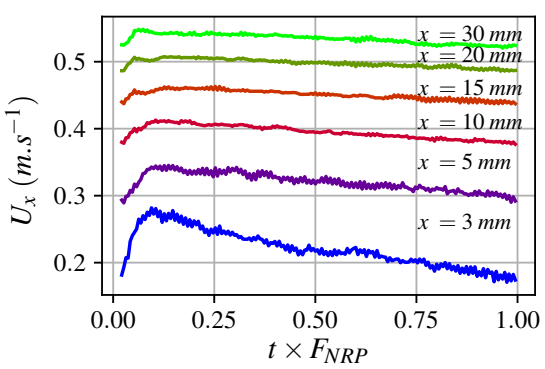

(a)

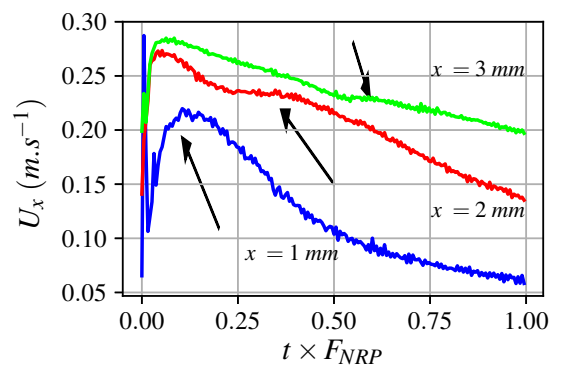

(b)

Figure 9. (a) Axial velocity $U_{x}$ at several $x$ locations averaged over 50 impulsions with $F_{N R P}=100 \mathrm{~Hz}$. Data has been obtained with the same set of figure 8 (a) and have been denoised by using a running mean with a kernel of 3 points. (b) Axial velocity $U_{x}$ at several $x$ locations computed over 50 impulsions and data has been obtained with the set of figure 8(b). Arrows highlight the acceleration corresponding to flow advection.

In order to have a better view near the microplasma, we used a long-range microscope instead of the objective to get a magnified velocity field. Note that the laser sheet was about $1 \mathrm{~mm}$ thick, so the field height and width are no longer very large compared to that of the laser sheet. We reported on figure 8(b) the magnified velocity field in the XY plane.

We acquired 8192 snapshots of the velocity fields to obtain figure 8 at frequency $F_{P I V}=16 \mathrm{kHz}$. By using these snapshots, we computed an average phase over a period sliced into $16000 / 100=160$ phases and averaged over $8192 / 160 \approx 51$ discharge events. The axial velocity is plotted for different positions on the central axis on figure 9(a) for the large field and on figure 9(b) for the magnified field. The discharge ignition is positioned at $t=0 \mathrm{~ms}$. On figure $9(\mathrm{a})$, a flow acceleration followed by a smooth velocity decrease are observed almost simultaneously at every reported value of $x$.

A similar behavior is observed on figure 9(b). A second bump indicated by arrows is visible and appears later. The shift in time between the curves for $x=1 \mathrm{~mm}, 2 \mathrm{~mm}$ 
and $3 \mathrm{~mm}$ indicates the advection of the velocity peak produced near the microplasma at $t \times F_{N R P}=0$. This second bump is no longer visible for $x=3 \mathrm{~mm}$ due to the high diffusion of momentum in viscous flows.

This low frequency case has shown that a significant acceleration is applied near the microplasma but also farther. This occurs between $t \times F_{N R P}=0$ to $t \times F_{N R P}=0.1$ $(t \in[0 \mathrm{~ms}, 1 \mathrm{~ms}])$ in the entire space between the microplasma and the plate. As in the case of $F_{N R P}=8 \mathrm{kHz}$, the long-range EHD force explains why the maximum velocity is found to be far from the microplasma. However the maximum acceleration, and thus the maximum EHD force, is applied near the microplasma. Thus, the physical mechanism of this acceleration phenomenon should be very fast compared to the microplasma period $(10 \mathrm{~ms})$. The characteristic pressure waves produced by NRP sparks can move at a velocity of $340 \mathrm{~m} \mathrm{~s}^{-1}$ in open air but the reported acceleration does not have an ovoid shape around the microplasma. It then should be due to ions which are moving at a mean velocity of $v_{i}=80 \mathrm{~m} \mathrm{~s}^{-1}$ and cross the $40 \mathrm{~mm}$ within $0.5 \mathrm{~ms}$ (corresponding to $t \times F_{N R P}=0.05$ on figure 9 ).

The fast-acting, long-range EHD force due to ion drift for the low-frequency case should also occur in the flow presented in figure 7. The ions are moving faster than the fluid and can accelerate the fluid before the jet front. By using low PRF with fast PIV, it is possible to obtain the clear time profiles of each acceleration event (figure 9).

\section{Conclusion}

We have reported on the use of a nanosecond repetitively pulsed microplasma to produce ionic wind in air at atmospheric pressure when a third electrode is used to provide the DC field to drive EHD flow. We have discussed first the main flow characteristics obtained with $F_{N R P}=8 \mathrm{kHz}, V_{\text {plate }}=-14 \mathrm{kV}$ and $d_{\text {gap }}=200 \mu \mathrm{m}$. The 
obtained flow velocity obtained with our geometry was about $2 \mathrm{~m} \mathrm{~s}^{-1}$ with a power of about $1 \mathrm{~W}$. These characteristics are on the order of magnitude of conventional discharges used to produce ionic wind such as corona discharges or DBDs. We showed that the flow field depends strongly on the magnitude of $V_{\text {plate }}$ but not on its polarity, indicating that the microplasma can produce both positive and negative ions that survive long enough to generate EHD in a large volume.

The use of the high-frequency PIV measurements enable the investigation of the transient properties of this flow. We observed the formation of the steady-state flow with the two accelerations operating at slow and fast time scales, corresponding to flows driven by advection and ion drift, respectively. The steady state flow at $F_{N R P}=8 \mathrm{kHz}$ is very similar to the behavior of an unsteady inertial jet with the advection of the jet front. However, by decreasing $F_{N R P}$ to $100 \mathrm{~Hz}$, the advective jet front is no longer visible very far from microplasma (at $x>5 \mathrm{~mm}$ ). At these distances, fluid motion is due instead to ion drift-based acceleration, a mechanism which acts at long range.

Several future prospects are expected. Those results highlights the flexibility to act upon the produced flow with precise timing. The surface covered by the microplasma is small (hundreds of microns) but we have showed that it can affect large areas by adding the third electrode. Furthermore, microplasmas can be used as arrays to affect larger areas.

When the charge production and charge transport are decoupled, numerical modeling can be used to design a microplasma actuator which can address specific problems where EHD flows are needed.

EHD theory and an experiment involving a simplified geometry (2D) could be easily compared. These properties can be used to enhance the velocity, or to expand 
or concentrate the produced flow.

Flow velocities and the average power were comparable with other discharges types. However, the high electric field and expected electron number density in this type of discharge can result in the generation of chemical species which cannot be obtained by corona discharges or DBD. Furthermore, the high electron number density provide by the NRP microplasma does not generate faster EHD flows than coronas or DBDs discharges with our geometry. Therefore, there is no evidence that EHD flows properties will be enhanced only by increasing the maximum electron number density of the microdischarge. Also, independent of charge density, charge transport in the EHD flow zone is critical. Further studies should focus on the ratio of the charges collected on the plate versus the charges produced by the microplasma, e. $g$. by measuring the current flowing through the third electrode.

Finally, it may be possible to obtain similar properties using a transient spark, whose generation involves simpler electronics. Transient sparks have already been shown to have similar characteristics as the discharge we used in this study in terms of the high-electron number density and high-conduction current (Janda et al. 2014).

\section{Acknowledgements}

Thomas Orrière has been supported by a $\mathrm{PhD}$ fellowship from the Ministère de l'Education Nationale, de l'Enseignement Supérieur et de la Recherche. We also gratefully acknowledge financial support from the Agence Nationale de la Recherche programs JCJC PLASMAFACE (ANR-15-CE06-0007-01) and INOPLAS (ANR-13ASTR-0015-01), the 'Investissements d'Avenir' program LABEX INTERACTIFS (ANR-11-LABX-0017-01) of the French government, the CPER program of the PoitouCharentes region, and the European Union (FEDER/FSE/FEADER programs). 
Electric wind generation by nanosecond repetitively pulsed microplasmas

\section{Bibliography}

Awad M B \& Castle G S P 1975 Journal of the Air Pollution Control Association 25(4), 369-374.

URL: http://www.tandfonline.com/doi/abs/10.1080/00022470.1975.10470092

Bayoda K D, Benard N \& Moreau E 2015 Journal of Applied Physics 118(6), 63301.

URL: http://scitation.aip.org/content/aip/journal/jap/118/6/10.1063/1.4927844

Becker K H, Schoenbach K H \& Eden J G 2006 Journal of Physics D: Applied Physics 39(3), R55$-\mathrm{R} 70$.

URL: http://stacks.iop.org/0022-3727/39/i=3/a=R01?key=crossref.d18fcd57c15de5558e13abfbea44bb2f

Boeuf J P \& Pitchford L C 2005 Journal of Applied Physics 97(10), 103307.

URL: https://aip-scitation-org.ressources.univ-poitiers.fr/doi/10.1063/1.1901841

Bölke O, Lacoste D A \& Moeck J P 2018 Journal of Physics D: Applied Physics 51(30), 305203.

URL: http://stacks.iop.org/0022-3727/51/i=30/a=305203?key=crossref.f3b93f9e421b629348411bc4a3da2fb5

Chen J \& Davidson J H 2002 Plasma Chemistry and Plasma Processing 22(4), 495-522.

URL: http://link.springer.com/10.1023/A:1021315412208

Christenson E A \& Moller P S 1967 AIAA Journal 5(10), 1768-1773.

URL: $h$ ttps://doi.org/10.2514/3.4302

Colas D F, Ferret A, Pai D Z, Lacoste D A \& Laux C O 2010 Journal of Applied Physics 108(10), 103306.

URL: http://aip.scitation.org/doi/10.1063/1.3514131

Corke T C, Enloe C L \& Wilkinson S P 2010 Annual Review of Fluid Mechanics 42(1), 505-529.

URL: http://www.annualreviews.org/doi/10.1146/annurev-fluid-121108-145550

Darny T, Pouvesle J M, Fontane J, Joly L, Dozias S \& Robert E 2017 Plasma Sources Science and Technology 26(10), 105001.

URL: http://stacks.iop.org/0963-0252/26/i=10/a=105001?key=crossref.465f2174d642e625e451323c4e310115

Defoort E, Bellanger R, Moreau E, Batiot-Dupeyrat C \& Tatibouet J M 2018 in '11ème conférence de la Société Française d'Electrostatique, SFE 2018, Grenoble'.

Go D B, Garimella S V, Fisher T S \& Mongia R K 2007 Journal of Applied Physics 102(5), 053302.

URL: https://doi.org/10.1063/1.2776164

Huang B D, Zhu X M, Takashima K \& Pu Y K 2013 Journal of Physics D: Applied Physics 46(46), 464011. 
Electric wind generation by nanosecond repetitively pulsed microplasmas

URL: $h t t p: / /$ stacks.iop.org/0022-3727/46/i=46/a=464011

Janda M, Martišovitš V, Hensel K, Dvonč L \& Machala Z 2014 Plasma Sources Science and Technology 23(6), 65016 .

URL: http://stacks.iop.org/0963-0252/23/i=6/a=065016

Johnson M J \& Go D B 2017 Plasma Sources Science and Technology 26(10), 103002.

URL: http://stacks.iop.org/0963-0252/26/i=10/a=103002

Johnson M J, Tirumala R \& Go D B 2015 Journal of Electrostatics 74, 8 - 14.

URL: http://www.sciencedirect.com/science/article/pii/S0304388614001168

Kogelschatz U, Eliasson B \& Egli W 1997 Le Journal de Physique IV 7(C4), C4-47.

Lacoste D A, Xiong Y, Moeck J P, Chung S H, Roberts W L \& Cha M S 2017 Proceedings of the Combustion Institute 36(3), 4183-4192.

URL: http://www.sciencedirect.com/science/article/pii/S1540748916300347

Lawton J \& Weinberg F J 1969 Electrical aspects of combustion Clarendon Press Oxford.

Macheret S O, Shneider M N \& Murray R C 2006 Physics of Plasmas (1994-present) 13(2), 23502.

URL: http://scitation.aip.org/content/aip/journal/pop/13/2/10.1063/1.2172926

Mariotti D \& Sankaran R M 2010 Journal of Physics D: Applied Physics 43(32), 323001.

URL: $h t t p: / / s t a c k s . i o p . o r g / 0022-3727 / 43 / i=32 / a=323001$

Martynenko A \& Kudra T 2016 Trends in Food Science \& Technology 54, 63 - 73.

URL: http://www.sciencedirect.com/science/article/pii/S0924224416300681

Moreau E 2007 Journal of Physics D: Applied Physics 40(3), 605.

URL: http://stacks.iop.org/0022-3727/40/i=3/a=S01

Moreau E, Audier P \& Benard N 2018 Journal of Electrostatics 93, 85 - 96.

URL: http://www.sciencedirect.com/science/article/pii/S0304388617303315

Moreau E, Audier P, Orriere T \& Benard N 2019 Journal of Applied Physics 125(13), 133303.

URL: $h t t p s: / /$ doi.org/10.1063/1.5056240

Moreau E, Benard N, Lan-Sun-Luk J D \& Chabriat J P 2013 Journal of Physics D: Applied Physics 46(47), 475204 .

URL: http://stacks.iop.org/0022-3727/46/i=47/a=475204

Moreau E, Louste C \& Touchard G 2008 Journal of Electrostatics 66(1-2), 107-114.

URL: https://linkinghub.elsevier.com/retrieve/pii/S0304388607001131

Nishida H, Nakai K \& Matsuno T 2017 AIAA Journal 55(6), 1852-1861. 
Electric wind generation by nanosecond repetitively pulsed microplasmas

URL: https://arc.aiaa.org/doi/10.2514/1.J055560

Nishida H, Nonomura T \& Abe T 2014 Journal of Applied Physics 115(13), 133301.

URL: http://aip.scitation.org/doi/10.1063/1.4870384

Orrière T, Moreau E \& Pai D Z 2018 Journal of Physics D: Applied Physics 51(49), 494002.

URL: http://stacks.iop.org/0022-3727/51/i=49/a=494002

Pancheshnyi S V, Lacoste D A, Bourdon A \& Laux C O 2006 IEEE Transactions on Plasma Science 34(6), 2478-2487.

URL: http://ieeexplore.ieee.org/lpdocs/epic03/wrapper.htm? arnumber=4032901

Pilla G, Galley D, Lacoste D A, Lacas F, Veynante D \& Laux C O 2006 IEEE Transactions on Plasma Science 34(6), 2471-2477.

Plank T, Jalakas A, Aints M, Paris P, Valk F, Viidebaum M \& Jõgi I 2014 Journal of Physics D: Applied Physics 47(33), 335205.

URL: $h t t p: / / s t a c k s . i o p . o r g / 0022-3727 / 47 / i=33 / a=335205$

Popov N A 2016 Plasma Sources Science and Technology 25(4), 44003.

URL: http://stacks.iop.org/0963-0252/25/i=4/a=044003

Radzig D A A \& Smirnov P B M 1985 in 'Reference Data on Atoms, Molecules, and Ions' number 31 in 'Springer Series in Chemical Physics' Springer Berlin Heidelberg pp. 429-438.

URL: http://link.springer.com/chapter/10.1007/978-3-642-82048-9_14

Robert E, Sarron V, Darny T, Riès D, Dozias S, Fontane J, Joly L \& Pouvesle J M 2014 Plasma Sources Science and Technology 23(1), 012003.

Robinson M 1961 Transactions of the American Institute of Electrical Engineers, Part I: Communication and Electronics 80(2), 143-150.

URL: http://ieeexplore.ieee.org/document/6373091/

Robinson M 1962 American Journal of Physics 30(5), 366-372.

URL: http://scitation.aip.org/content/aapt/journal/ajp/30/5/10.1119/1.1942021

Rusterholtz D L, Lacoste D A, Stancu G D, Pai D Z \& Laux C O 2013 Journal of Physics D: Applied Physics 46(46), 464010.

URL: http://stacks.iop.org/0022-3727/46/i=46/a=464010

Seydou Moumouni A 2016 Etude expérimentale des effets hydrodynamiques des décharges nanosecondes répétitives pulsées (NRP) dans l'interaction plasma-flamme $\mathrm{PhD}$ thesis.

URL: http://www.theses.fr/2016ESMA0029 
Electric wind generation by nanosecond repetitively pulsed microplasmas

Tirumala R \& Go D B 2011 IEEE Transactions on Dielectrics and Electrical Insulation 18(6), 18541863.

Van der Horst R M, Verreycken T, van Veldhuizen E M \& Bruggeman P J 2012 Journal of Physics D: Applied Physics 45(34), 345201.

URL: http://stacks.iop.org/0022-3727/45/i=34/a=345201

Willert C E, Mitchell D M \& Soria J 2012 Experiments in Fluids 53(2), 413-421.

URL: http://link.springer.com/10.1007/s00348-012-1297-1

Xu D A, Lacoste D A \& Laux C O 2015 Plasma Chemistry and Plasma Processing 36(1), 309-327.

URL: http://link.springer.com/article/10.1007/s11090-015-9680-3

Xu H, He Y, Strobel K L, Gilmore C K, Kelley S P, Hennick C C, Sebastian T, Woolston M R, Perreault D J \& Barrett S R H 2018 Nature 563(7732), 532-535.

URL: http://www.nature.com/articles/s41586-018-0707-9

Yehia A \& Mizuno A 2005 in 'Fourtieth IAS Annual Meeting. Conference Record of the 2005 Industry Applications Conference, 2005.' Vol. 3 IEEE pp. 1828-1832.

URL: http://ieeexplore.ieee.org/document/1518694/

Zhu X M, Walsh J L, Chen W C \& Pu Y K 2012 Journal of Physics D: Applied Physics 45(29), 295201.

URL: $h$ ttp://stacks.iop.org/0022-3727/45/i=29/a=295201?key=crossref.a51a2197433aad50ab95f12475e69be1

Zong H \& Kotsonis M 2018 Journal of Fluid Mechanics 837, 147-181.

URL: https://www.cambridge.org/core/product/identifier/S0022112017008552/type/journal_article

Zouzou N \& Moreau E 2011 Journal of Physics D: Applied Physics 44(28), 285204.

URL: http://stacks.iop.org/0022-3727/44/i=28/a=285204

\section{Appendix A. $d_{g a p}$ variation}

In this section, we study the variation of the gap distance of the microplasma. In particular, we compare the microplasma regime with a discharge at the mm-scale. We used a PIV system different from the previously reported setup to acquire the mean velocity field. In addition, we used a Schlieren imaging experiment to compare the shape of the flow with the PIV measurement. This technique does not require seed particles and can work in open air. We mention in the following only the 


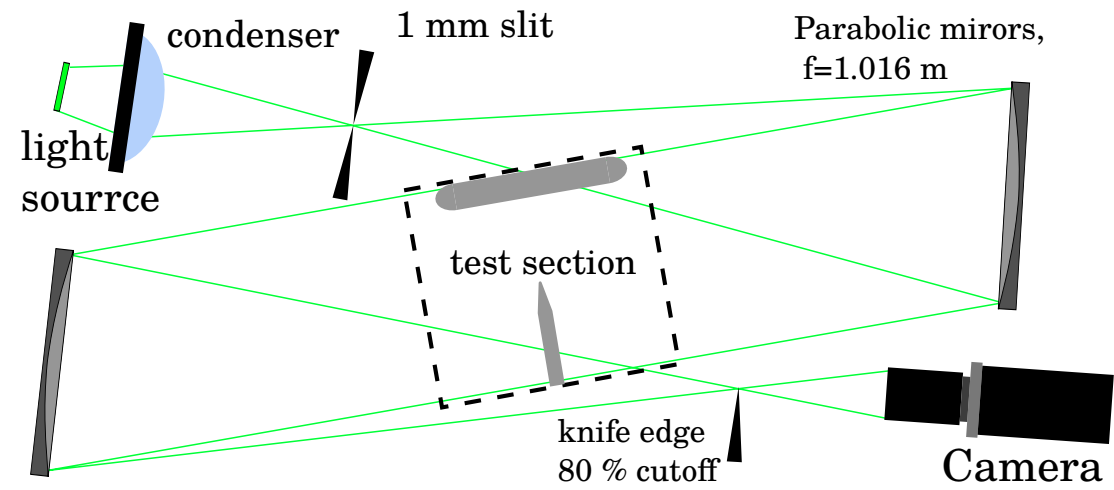

Figure A1. Schematic diagram of the setup for Schlieren imaging

differences between the previous setup and our referred experimental conditions in section Appendix A.1.

Appendix A.1. PIV system

A $8 \mathrm{~Hz}$ PIV system has been used with a camera (Lavision, Flowmaster 3S) equipped with a $200 \mathrm{~mm}$ objective (AF Micro-NIKKOR $200 \mathrm{~mm}$ f/4 DIF-ED Nikon) and a (Ndyag Laser QUANTEL, model Twins-Ultra). The obtained field of view was $54 \times 43 \mathrm{~mm}$. Each mean velocity field required 500 snapshots of the velocity field, and thus 500 dual images were recorded at a frequency $F_{P I V}=3.9 \mathrm{~Hz}$. Images were treated with Davis 7.2 software with $32 \times 32$ pixels interrogation windows with a $50 \%$ overlap. The obtained field was then a $80 \times 64$ vector grid. Finally the plate was a circle of aluminum tape with a diameter of $40 \mathrm{~mm}$. The plate distance was $d_{\text {plate }}=40 \mathrm{~mm}$ and the applied potential was $V_{\text {plate }}=-16 \mathrm{kV}$.

\section{Appendix A.2. Schlieren imaging}

As shown on figure A1, we used a Z-type system with two spherical mirrors $50 \mathrm{~mm}$ in diameter and $1.016 \mathrm{~m}$ in focal length. A high-power diode (Luminus CBT-120, 
green) produced $1 \mu$ s light pulses. We used the electrical circuit described by (Willert et al. 2012). The entrance slit was adjusted to $1 \mathrm{~mm}$-height, and the knife-edge was positioned to cutoff $80 \%$ of the light. A camera (JAI model CV-M2) with a $1600 \times 1200$ pixel CCD sensor recorded the images. To minimize jitter, we used the PIV mode of the camera, taking dual images at a frequency of $15 \mathrm{~Hz}$.

To fit entire field on the sensor, we used $d_{\text {plate }}=35 \mathrm{~mm}$ because the field width was $37 \mathrm{~mm}$. To match the experimental conditions of the PIV measurements, we used $V_{\text {plate }}=-14 \mathrm{kV}$.

Appendix A.3. Results

Figures A2 shows Schlieren and PIV measurements for three different $d_{\text {gap }}$ distances : $50 \mu \mathrm{m}, 200 \mu \mathrm{m}$ and $2 \mathrm{~mm}$. Discharges are repeated at a frequency $F_{N R P}=8 \mathrm{kHz}$, and the ratio $U_{\text {plasma }} / d_{\text {gap }}$ has been kept constant.

We can see on figures A2(a-f) that the variation of contrast representative of the gas density increases with the gap distance. Furthermore the signal of the pressure wave (figures A2(a-c)) following the generation of a spark discharge also increases with the gap distance. 


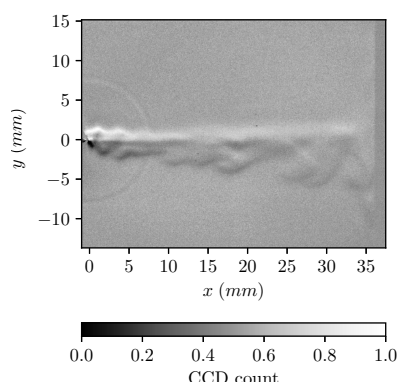

(a)
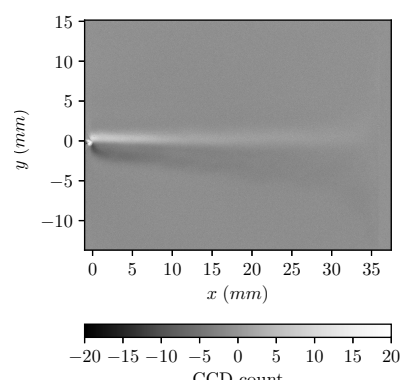

(d)

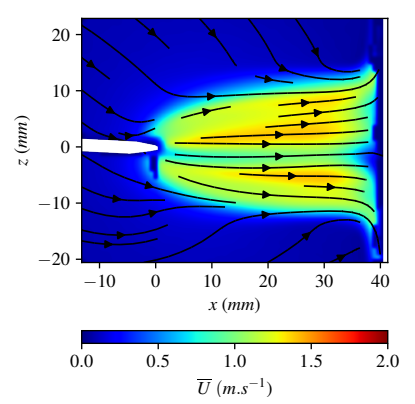

$(\mathrm{g})$

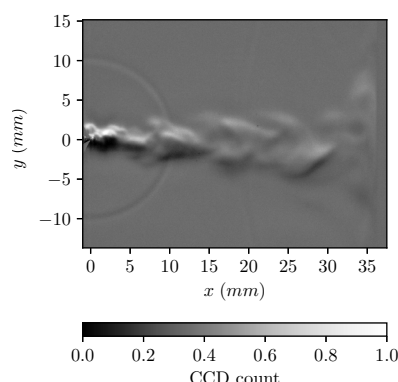

(b)

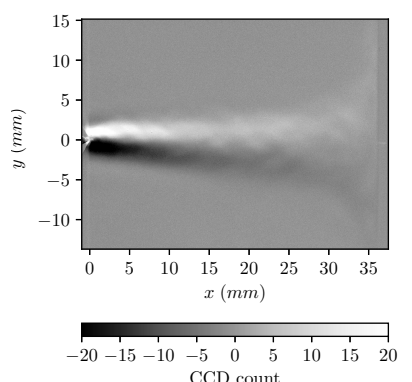

(e)

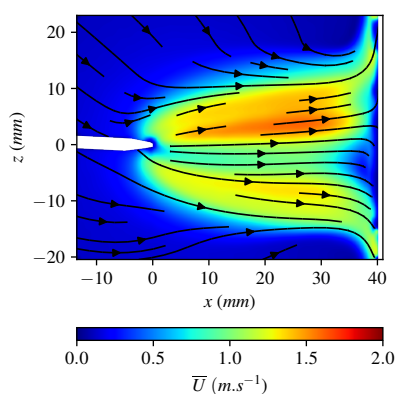

(h)

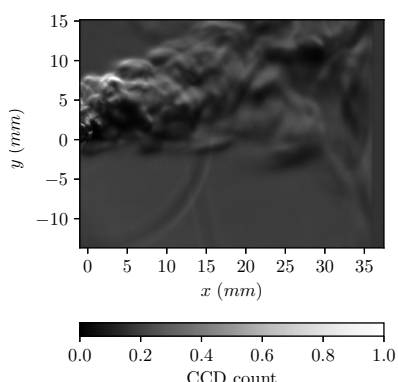

(c)

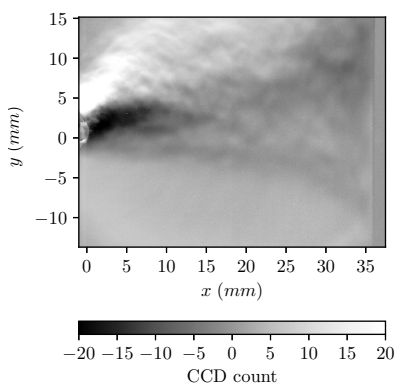

(f)

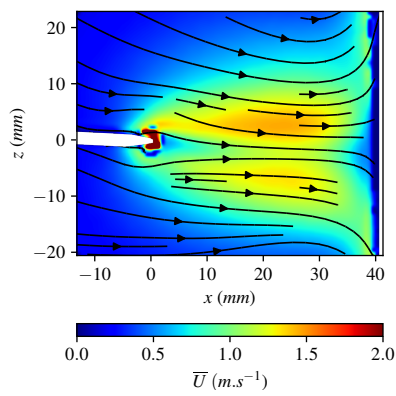

(i)

Figure A2. Snapshots of the Schlieren imaging for (a) $d_{\text {gap }}=50 \mu \mathrm{m}$, (b) $d_{\text {gap }}=$ $200 \mu \mathrm{m}$, (c) $d_{\text {gap }}=2 \mathrm{~mm}$, averaged schlieren images over 200 snapshots for (d) $d_{\text {gap }}=50 \mu \mathrm{m},(\mathrm{e}) d_{\text {gap }}=200 \mu \mathrm{m}$, (f) $d_{\text {gap }}=2 \mathrm{~mm}$ and average velocity magnitude field for $(\mathrm{g}) d_{\text {gap }}=50 \mu \mathrm{m},(\mathrm{h}) d_{\text {gap }}=200 \mu \mathrm{m}$, (i) $d_{\text {gap }}=2 \mathrm{~mm}$. Schlieren photography has been taken in the XY plane while the flow velocity fields have been taken in the XZ plane.

Then we observe from the flow velocity fields (figures A2(g-i)) that the maximum flow velocity does not significantly change with increasing gap distance, while the diameter of the generated flow weakly increases. Thus the dissipated power by the forced convection from EHD should not be significantly different, even though the deposited energy increases linearly with gap distance. The additional energy is 
spent thermally. On figure A2(c) and (f), we further see buoyancy effects in the XY plane. Thus in our studied range, only the mm-scale spark discharge exhibits natural convection due to gas heating by the discharge. When no EHD force is present, the natural convection is directed vertically from the electrodes (Xu et al. 2015). The flow velocity field in the $\mathrm{XZ}$ plane (figure A2(i)) remains unchanged in its topology. The distance $d_{g a p}$ could be an interesting parameter for tuning the relative influence of EHD or gas heating on the flow field. 